\title{
Absence of Global Solutions for a Fractional in Time and Space Shallow-Water System
}

\author{
Mohamed Jleli $^{1}$, Mokhtar Kirane ${ }^{2,3, *}$ and Bessem Samet ${ }^{1}$ \\ 1 Department of Mathematics, College of Science King Saud University, P.O. Box 2455, \\ Riyadh 11451, Saudi Arabia; jleli@ksu.edu.sa (M.J.); bsamet@ksu.edu.sa (B.S.) \\ 2 LaSIE, Pôle Sciences et Technologies, Université de La Rochelle, Avenue Michel Crépeau, \\ 17031 La Rochelle, France \\ 3 Peoples' Friendship University of Russia (RUDN University), 6 Miklukho-Maklaya Street, \\ 117198 Moscow, Russia \\ * Correspondence: mokhtar.kirane@univ-lr.fr
}

Received: 12 October 2019; Accepted: 15 November 2019; Published: 18 November 2019

\begin{abstract}
An initial boundary value problem for a fractional in time and space shallow-water system involving $\psi$-Caputo fractional derivatives of different orders is considered. Using the test function method, sufficient criteria for the absence of global in time solutions of the system are obtained.
\end{abstract}

Keywords: global solutions; fractional in time and space shallow-water system; $\psi$-Caputo fractional derivative

MSC: 35B44; 26A33

\section{Introduction}

We consider the fractional in time and space shallow-water system

$$
\begin{cases}\partial_{0 \mid t}^{\alpha, \psi} \eta+\partial_{0 \mid x}^{\beta, \psi}(\eta u) & =0, \quad t>0,0<x<L, \\ \frac{1}{2}\left[\frac{1}{\psi^{\prime}(t)} \partial_{t}(\eta u)+\partial_{0 \mid t}^{\alpha, \psi}(\eta u)\right]+\partial_{0 \mid x}^{\beta, \psi}\left(\eta u^{2}\right)+\partial_{0 \mid x}^{\beta, \psi}\left(\eta^{2}\right) & =0, \quad t>0,0<x<L\end{cases}
$$

with

$$
(u(0, \cdot), \eta(0, \cdot))=\left(u_{0}, \eta_{0}\right)
$$

and

$$
\eta(\cdot, 0)=\eta(\cdot, L) \equiv 0 ;
$$

here $\eta=\eta(t, x), u=u(t, x), L>0,0<\alpha, \beta<1, \psi \in C^{1}([0, \infty)), \lim _{x \rightarrow \infty} \psi(x)=+\infty, \psi^{\prime}(x)>0$, $x \geq 0, \partial_{0 \mid t}^{\alpha, \psi}$ is the $\psi$-Caputo derivative in time of fractional order $\alpha$ and $\partial_{0 \mid x}^{\beta, \psi}$ is the $\psi$-Caputo derivative in space of fractional order $\beta$. Using the test function method [1], we get sufficient criteria for which problem (1)-(2)-(3) has no global solutions in time.

The considered problem is a fractional version of the shallow-water system

$$
\begin{cases}\partial_{t} \eta+\partial_{x}(\eta u) & =0, \quad t>0,0<x<L \\ \partial_{t}(\eta u)+\partial_{x}\left(\eta u^{2}\right)+\partial_{x}\left(\eta^{2}\right) & =0, \quad t>0,0<x<L\end{cases}
$$

which models the motion of an incompressible fluid in a gravitational field when the fluid height above the channel bottom is small with respect to the characteristic flow length. Here $u$ is the velocity of the fluid particle and $\eta$ is the height of the fluid above the horizontal flat bottom [2-4]. 
In [2], Korpusov and Yushkov derived sufficient criteria for the non-existence of global in time solutions of problem (4) under different types of boundary conditions. In particular, under the boundary conditions (3), they proved that if for some $0<T_{0}<\infty$, the problem admits a solution $(u, \eta) \in C^{1}\left(\left[0, T_{0}\right] \times[0, L]\right) \times C^{1}\left(\left[0, T_{0}\right] \times[0, L]\right)$, and

$$
\int_{0}^{L} x \eta_{0}(x) u_{0}(x) d x>0,
$$

then there exist no solutions on intervals larger than $\left[0, T_{\infty}\right]$, where

$$
T_{\infty}=\frac{L^{2} \int_{0}^{L} \eta_{0}(x) d x}{\int_{0}^{L} x \eta_{0}(x) u_{0}(x) d x} .
$$

It was shown in many published works that the theory of fractional calculus provides useful tools for modeling various phenomena from physics (see e.g., [5-8]). Specifically, it was found that fractional order models of many real-world phenomena are more adequate than the classical integer order models. This fact motivated researchers to take an interest in the study of fractional in time and/or space evolution equations. In particular, the study of analytic and numerical solutions of fractional shallow-water equations was investigated by many authors (see e.g., [5,9-12]). For the study of existence and non-existence of global solutions for fractional in time and/or space evolution equations, we refer to [13-15] and references therein.

Motivated by the above contributions, the study of the absence of global in time solutions for problem (1)-(2)-(3) is investigated in this work. In the considered problem, we use $\psi$-Caputo fractional derivative (in time and space) [16], which depends of a function $\psi \in C^{1}([0, \infty))$. In the special case $\psi(t)=t$, the considered fractional operator reduces to Caputo fractional derivative. Let us mention that in this paper we are concerned essentially with the mathematical study of problem (1)-(2)-(3). For the physical interpretation of this model, we are not able to check if it is more adequate than the standard model (4)-(2)-(3). For a such study, some physical experiments and numerical simulations are needed; this is not the goal of this paper. Nevertheless, let us notice that Tao in [17] proposed a possible scenario for obtaining blowing-up solutions of the Navier-Stokes system; he showed that it is possible for a body of fluid to form a sort of computer, which can build a self-replicating fluid robot that keeps transferring its energy to smaller and smaller copies of itself until the fluid "blows up." He tried to devise a system that would incorporate a delay at each step-a sort of timer that would push the energy cleanly from one size scale to the next at just the right moment (according to Erica Klarreich, A Fluid New Path in Grand Math Challenge, Quantamagazine, 24 February 2014). From here, one can speculate any form of delay in time or space for fluid dynamical systems.

In Section 2, we provide some preliminary results that will be needed afterwards. A key lemma is established in Section 3. In the next section, we present and establish our principal results. Specifically, we first establish a mass conservation law for problem (1)-(2)-(3). Next, we obtain sufficient criteria for which the considered problem has no global in time solutions.

\section{Preliminaries}

Let $c_{1}, c_{2} \in \mathbb{R}, c_{1}<c_{2}, \mathcal{R} \in L^{1}\left(c_{1}, c_{2}\right)$ and $\mu>0$. The Riemann-Liouville fractional integrals of order $\mu$ of $\mathcal{R}$ are given by (see e.g., [18])

$$
I_{\mathcal{c}_{1}}^{\mu} \mathcal{R}(x)=[\Gamma(\mu)]^{-1} \int_{\mathcal{c}_{1}}^{x}(x-\sigma)^{\mu-1} \mathcal{R}(\sigma) d \sigma
$$

and

$$
I_{\mathcal{C}_{2}}^{\mu} \mathcal{R}(x)=[\Gamma(\mu)]^{-1} \int_{x}^{\mathcal{C}_{2}}(\sigma-x)^{\mu-1} \mathcal{R}(\sigma) d \sigma,
$$

for a.e. $x \in\left[c_{1}, c_{2}\right]$, where $\Gamma$ denotes the gamma function. 
Let $\psi$ be a $C^{1}$ function in $[0, \infty)$ satisfying

$$
\lim _{x \rightarrow \infty} \psi(x)=+\infty \quad \text { and } \quad \psi^{\prime}(x)>0, \quad x \geq 0 .
$$

Please note that under the above conditions, the function $\psi:[0, \infty) \rightarrow[\psi(0), \infty)$ is bijective. Let $\tau>0$ and $\mathcal{R} \in L^{1}\left((0, \tau), \psi^{\prime}(\sigma) d \sigma\right)$, i.e.,

$$
\int_{0}^{\tau}|\mathcal{R}(\sigma)| \psi^{\prime}(\sigma) d \sigma<\infty
$$

The $\psi$-fractional integrals of order $\mu$ of $\mathcal{R}$ are given by (see [16])

$$
I_{0}^{\mu, \psi} \mathcal{R}(x)=[\Gamma(\mu)]^{-1} \int_{0}^{x}(\psi(x)-\psi(\sigma))^{\mu-1} \psi^{\prime}(\sigma) \mathcal{R}(\sigma) d \sigma
$$

and

$$
I_{\tau}^{\mu, \psi} \mathcal{R}(x)=[\Gamma(\mu)]^{-1} \int_{x}^{\tau}(\psi(\sigma)-\psi(x))^{\mu-1} \psi^{\prime}(\sigma) \mathcal{R}(\sigma) d \sigma,
$$

for a.e. $x \in[0, \tau]$.

If $\mathcal{R} \in C([0, \tau])$, then $I_{0}^{\mu, \psi} \mathcal{R}, I_{\tau}^{\mu, \psi} \mathcal{R} \in C([0, \tau])$ and $I_{0}^{\mu, \psi} \mathcal{R}(0)=I_{\tau}^{\mu, \psi} \mathcal{R}(\tau)=0$.

Lemma 1. For $\mathcal{R} \in L^{1}\left((0, \tau), \psi^{\prime}(\sigma) d \sigma\right)$, it holds

$$
\left(I_{0}^{\mu, \psi} \mathcal{R}\right)(x)=\left(I_{\psi(0)}^{\mu} \mathcal{R} \circ \psi^{-1}\right)(\psi(x)) \text {, a.e. } x \in[0, \tau]
$$

and

$$
\left(I_{\tau}^{\mu, \psi} \mathcal{R}\right)(x)=\left(I_{\psi(\tau)}^{\mu} \mathcal{R} \circ \psi^{-1}\right)(\psi(x)), \quad \text { a.e. } x \in[0, \tau],
$$

where $\circ$ stands for the composition of mappings.

Proof. For a.e. $x \in[0, \tau]$, one has

$$
\begin{aligned}
\left(I_{0}^{\mu, \psi} \mathcal{R}\right)(x) & =[\Gamma(\mu)]^{-1} \int_{0}^{x}(\psi(x)-\psi(\sigma))^{\mu-1} \psi^{\prime}(\sigma) \mathcal{R}(\sigma) d \sigma \\
& =[\Gamma(\mu)]^{-1} \int_{\psi(0)}^{\psi(x)}(\psi(x)-t)^{\mu-1} \mathcal{R}\left(\psi^{-1}(t)\right) d t \\
& =\left(I_{\psi(0)}^{\mu} \mathcal{R} \circ \psi^{-1}\right)(\psi(x)),
\end{aligned}
$$

which proves (6). Proceeding as above, one obtains (7).

Lemma 2 (see e.g., [18]). Let $(\mathcal{R}, \mathcal{S}) \in L^{1}(a, b) \times C([a, b])$. Then

$$
\int_{a}^{b} \mathcal{R}(t)\left(I_{a}^{\mu} \mathcal{S}\right)(t) d t=\int_{a}^{b} \mathcal{S}(t)\left(I_{b}^{\mu} \mathcal{R}\right)(t) d t
$$

Lemma 3. Let $(\mathcal{R}, \mathcal{S}) \in L^{1}\left((0, \tau), \psi^{\prime}(\sigma) d \sigma\right) \times C([0, \tau])$. Then

$$
\int_{0}^{\tau} \mathcal{R}(\sigma)\left(I_{0}^{\mu, \psi} \mathcal{S}\right)(\sigma) \psi^{\prime}(\sigma) d \sigma=\int_{0}^{\tau} \mathcal{S}(\sigma)\left(I_{\tau}^{\mu, \psi} \mathcal{R}\right)(\sigma) \psi^{\prime}(\sigma) d \sigma .
$$


Proof. Using (6), one obtains

$$
\begin{aligned}
\int_{0}^{\tau} \mathcal{R}(\sigma)\left(I_{0}^{\mu, \psi} \mathcal{S}\right)(\sigma) \psi^{\prime}(\sigma) d \sigma & =\int_{0}^{\tau} \mathcal{R}(\sigma)\left(I_{\psi(0)}^{\mu} \mathcal{S} \circ \psi^{-1}\right)(\psi(\sigma)) \psi^{\prime}(\sigma) d \sigma \\
& =\int_{\psi(0)}^{\psi(\tau)} \mathcal{R}\left(\psi^{-1}(t)\right)\left(I_{\psi(0)}^{\mu} \mathcal{S} \circ \psi^{-1}\right)(t) d t
\end{aligned}
$$

Next, using Lemma 2, one deduces that

$$
\int_{0}^{\tau} \mathcal{R}(\sigma)\left(I_{0}^{\mu, \psi} \mathcal{S}\right)(\sigma) \psi^{\prime}(\sigma) d \sigma=\int_{\psi(0)}^{\psi(\tau)} \mathcal{S} \circ \psi^{-1}(t)\left(I_{\psi(\tau)}^{\mu} \mathcal{R} \circ \psi^{-1}\right)(t) d t .
$$

Hence, by (7), the desired result follows.

Let $\mathcal{R} \in C^{1}([0, \tau])$ and $0<\theta<1$. The $\psi$-Caputo fractional derivative of order $\theta$ of $\mathcal{R}$ is given by (see [16])

$$
\left(\partial_{0 \mid x}^{\theta, \psi} \mathcal{R}\right)(x)=\left(I_{0}^{1-\theta, \psi} \frac{\mathcal{R}^{\prime}}{\psi^{\prime}}\right)(x), \quad 0 \leq x \leq \tau
$$

i.e.,

$$
\left(\partial_{0 \mid x}^{\theta, \psi} \mathcal{R}\right)(x)=[\Gamma(1-\theta)]^{-1} \int_{0}^{x}(\psi(x)-\psi(\sigma))^{-\theta} \mathcal{R}^{\prime}(\sigma) d \sigma
$$

Lemma 4 (see [16]). Let $\mathcal{R} \in C^{1}([0, \tau])$ and $0<\theta<1$. One has

$$
I_{0}^{\theta, \psi}\left(\partial_{0 \mid x}^{\theta, \psi} \mathcal{R}\right)(x)=\mathcal{R}(x)-\mathcal{R}(0), \quad 0 \leq x \leq \tau
$$

\section{A Key Lemma}

The following lemma will be useful for proving our principal result.

Lemma 5. Let $0<\theta<1$ and $a>0$. Suppose that for some $0<T_{0}<\infty, J \in C^{1}\left(\left[0, T_{0}\right]\right)$ is a function satisfying $J(0)>0$ and

$$
\frac{1}{\psi^{\prime}(t)} J^{\prime}(t)+\left(\partial_{0 \mid t}^{\theta, \psi} J\right)(t) \geq a J^{2}(t), \quad 0<t<T_{0}
$$

Let

$$
T_{\infty}:=\sup \left\{\tau>0: J \in C^{1}([0, \tau)) \text { satisfies (9) for all } 0<t<\tau\right\}
$$

Then

$$
T_{0} \leq T_{\infty} \leq \psi^{-1}(\psi(0)+M(a, \theta))<\infty
$$

where

$$
M(a, \theta)=\sup \{X>0: f(X) \leq 0\}<\infty, \quad f(X)=\mathcal{B} X^{2-\theta}+\mathcal{C} X-\mathcal{D} X^{2-2 \theta}-1
$$

and

$$
\mathcal{B}=\frac{a}{\Gamma(4-\theta)} J(0), \mathcal{C}=\frac{a}{2} J(0), \mathcal{D}=\frac{1}{(3-2 \theta) \Gamma(3-\theta)^{2}}
$$

Proof. First, since $f(0)=-1<0$ and $f$ is continuous, $\{X>0: f(X) \leq 0\} \neq \varnothing$. Furthermore, since $B>0$ (because $J(0)>0$ ), on has $\lim _{X \rightarrow+\infty} f(X)=+\infty$. Hence, one deduces that $0<M(a, \theta)<\infty$.

Next, let $\tau>0$ be such that $J \in C^{1}([0, \tau))$ satisfies (9) for all $0<t<\tau$. Then, for all $0<T<\tau$, $J \in C^{1}([0, T])$ satisfies (9) for all $0<t<T$. Fix $0<T<\tau$ and introduce the function

$$
\varphi(t)=\kappa(0)^{-2} \kappa(t)^{2} \psi^{\prime}(t):=\mathcal{Z}(t) \psi^{\prime}(t)
$$


for all $0 \leq t \leq T$, where

$$
\kappa(t)=\psi(T)-\psi(t)
$$

Using (9), one obtains

$$
a \int_{0}^{T} J^{2}(t) \varphi(t) d t \leq \int_{0}^{T}\left(\partial_{0 \mid t}^{\theta, \psi} J\right)(t) \varphi(t) d t+\int_{0}^{T} J^{\prime}(t) \mathcal{Z}(t) d t .
$$

On the other hand, using (8) and Lemma 3, one has

$$
\begin{aligned}
\int_{0}^{T}\left(\partial_{0 \mid t}^{\theta, \psi} J\right)(t) \varphi(t) d t & =\int_{0}^{T}\left(I_{0}^{1-\theta, \psi} \frac{J^{\prime}}{\psi^{\prime}}\right)(t) \varphi(t) d t \\
& =\int_{0}^{T} J^{\prime}(t)\left(I_{T}^{1-\theta, \psi} \mathcal{Z}\right)(t) d t
\end{aligned}
$$

Integrating by parts, it holds

$$
\int_{0}^{T}\left(\partial_{0 \mid t}^{\theta, \psi} J\right)(t) \varphi(t) d t=J(T)\left(I_{T}^{1-\theta, \psi} \mathcal{Z}\right)(T)-J(0)\left(I_{T}^{1-\theta, \psi} \mathcal{Z}\right)(0)-\int_{0}^{T} J(t)\left(I_{T}^{1-\theta, \psi} \mathcal{Z}\right)^{\prime}(t) d t
$$

Using (5), an elementary calculation gives us that

$$
\left(I_{T}^{1-\theta, \psi} \mathcal{Z}\right)(t)=\frac{2}{\Gamma(4-\theta)} \kappa(0)^{-2} \kappa(t)^{3-\theta}
$$

and

$$
\left(I_{T}^{1-\theta, \psi} \mathcal{Z}\right)^{\prime}(t)=-\frac{2}{\Gamma(3-\theta)} \kappa(0)^{-2} \kappa(t)^{2-\theta} \psi^{\prime}(t),
$$

for all $0 \leq t \leq T$. Using (14) and (15), one deduces that

$$
\int_{0}^{T}\left(\partial_{0 \mid t}^{\theta, \psi} J\right)(t) \varphi(t) d t=-\frac{2}{\Gamma(4-\theta)} \kappa(0)^{1-\theta} J(0)-\int_{0}^{T} J(t)\left(I_{T}^{1-\theta, \psi} \mathcal{Z}\right)^{\prime}(t) d t .
$$

Again, integrating by parts, it holds

$$
\int_{0}^{T} J^{\prime}(t) \mathcal{Z}(t) d t=-J(0)-\int_{0}^{T} J(t) \mathcal{Z}^{\prime}(t) d t .
$$

Hence, it follows from (13), (17) and (18) that

$$
\mathcal{A} J(0)+a \int_{0}^{T} J^{2}(t) \varphi(t) d t \leq \int_{0}^{T}|J(t)|\left|\left(I_{T}^{1-\theta, \psi} \mathcal{Z}\right)^{\prime}(t)\right| d t+\int_{0}^{T}|J(t)|\left|\mathcal{Z}^{\prime}(t)\right| d t,
$$

where

$$
\mathcal{A}=\frac{2}{\Gamma(4-\theta)} \kappa(0)^{1-\theta}+1
$$

On the other hand, by Young's inequality with parameter $\frac{a}{2}>0$, one has

$$
\begin{aligned}
\int_{0}^{T}|J(t)|\left|\left(I_{T}^{1-\theta, \psi} \mathcal{Z}\right)^{\prime}(t)\right| d t & =\int_{0}^{T} \sqrt{a \varphi(t)}|J(t)| \frac{\left|\left(I_{T}^{1-\theta, \psi} \mathcal{Z}\right)^{\prime}(t)\right|}{\sqrt{a \varphi(t)}} d t \\
& \leq \frac{a}{2} \int_{0}^{T} J^{2}(t) \varphi(t) d t+\frac{1}{2 a} \int_{0}^{T} \frac{\left|\left(I_{T}^{1-\theta, \psi} \mathcal{Z}\right)^{\prime}(t)\right|^{2}}{\varphi(t)} d t
\end{aligned}
$$


Similarly, one gets

$$
\int_{0}^{T}|J(t)|\left|\mathcal{Z}^{\prime}(t)\right| d t \leq \frac{a}{2} \int_{0}^{T} J^{2}(t) \varphi(t) d t+\frac{1}{2 a} \int_{0}^{T} \frac{\left|\mathcal{Z}^{\prime}(t)\right|^{2}}{\varphi(t)} d t .
$$

Combining (19)-(21), it comes that

$$
2 a \mathcal{A} J(0) \leq \int_{0}^{T} \frac{\left|\left(I_{T}^{1-\theta, \psi} \mathcal{Z}\right)^{\prime}(t)\right|^{2}}{\varphi(t)} d t+\int_{0}^{T} \frac{\left|\mathcal{Z}^{\prime}(t)\right|^{2}}{\varphi(t)} d t .
$$

Furthermore, using (12) and (16), one obtains

$$
\frac{\left|\left(I_{T}^{1-\theta, \psi} \mathcal{Z}\right)^{\prime}(t)\right|^{2}}{\varphi(t)}=\left[\frac{2}{\Gamma(3-\theta)}\right]^{2} \kappa(0)^{-2} \kappa(t)^{2-2 \theta} \psi^{\prime}(t),
$$

for all $0<t<T$, which yields

$$
\int_{0}^{T} \frac{\left|\left(I_{T}^{1-\theta, \psi} \mathcal{Z}\right)^{\prime}(t)\right|^{2}}{\varphi(t)} d t=\frac{1}{(3-2 \theta)}\left[\frac{2}{\Gamma(3-\theta)}\right]^{2} \kappa(0)^{1-2 \theta} .
$$

Similar calculations yield

$$
\int_{0}^{T} \frac{\left|\mathcal{Z}^{\prime}(t)\right|^{2}}{\varphi(t)} d t=4 \kappa(0)^{-1}
$$

It follows from (22)-(24) that

$$
2 a \mathcal{A} J(0) \leq \frac{1}{(3-2 \theta)}\left[\frac{2}{\Gamma(3-\theta)}\right]^{2} \kappa(0)^{1-2 \theta}+4 \kappa(0)^{-1},
$$

which yields

$$
f(\kappa(0)) \leq 0 .
$$

Therefore, one deduces that

$$
\kappa(0) \leq M(a, \theta) .
$$

Hence, it holds

$$
T \leq \psi^{-1}(\psi(0)+M(a, \theta)), \quad \text { for all } 0<T<\tau,
$$

which implies that

$$
\tau \leq \psi^{-1}(\psi(0)+M(a, \theta)),
$$

and (10) follows.

Remark 1. Taking $\psi(t)=t$ and the limit as $\theta \rightarrow 1^{-}$, (9) reduces to

$$
J^{\prime}(t) \geq \frac{a}{2} J^{2}(t), \quad 0<t<T_{0} .
$$

Hence, under the assumptions of Lemma 5, passing to the limit as $\theta \rightarrow 1^{-}$in (10), it holds

$$
T_{0} \leq T_{\infty} \leq \frac{2}{a J(0)},
$$

which is the same estimate as in ([19], Corollary 1). 


\section{Non-Existence of Global in Time Solutions for Problem (1)-(2)-(3)}

We assume that

(i) $0<\alpha, \beta<1, L>0$.

(ii) $\psi \in C^{1}([0, \infty)), \lim _{x \rightarrow \infty} \psi(x)=+\infty, \psi^{\prime}(x)>0, x \geq 0$.

(iii) $\eta_{0}, u_{0} \in C^{1}([0, L])$.

We first establish the following mass conservation law.

Proposition 1. Suppose that for some $0<T_{0}<\infty,(\eta, u) \in C^{1}\left(\left[0, T_{0}\right] \times[0, L]\right) \times C^{1}\left(\left[0, T_{0}\right] \times[0, L]\right)$, $\eta \geq 0$, is a solution of problem (1)-(2)-(3). Then

$$
\int_{0}^{L} \mathcal{K}_{L}(x)^{\beta-1} \eta(t, x) \psi^{\prime}(x) d x=\int_{0}^{L} \mathcal{K}_{L}(x)^{\beta-1} \eta_{0}(x) \psi^{\prime}(x) d x:=m_{0}, \quad 0 \leq t \leq T_{0}
$$

where

$$
\mathcal{K}_{L}(x)=\psi(L)-\psi(x)
$$

Proof. From the first equation in (1), one has

$$
-\partial_{0 \mid t}^{\alpha, \psi} \eta(t, x)=\partial_{0 \mid x}^{\beta, \psi}(\eta u)(t, x), \quad(t, x) \in\left(0, T_{0}\right] \times(0, L),
$$

whereupon

$$
-\partial_{0 \mid t}^{\alpha, \psi}\left(I_{0}^{\beta, \psi} \eta(t, \cdot)\right)(L)=\left(I_{0}^{\beta, \psi} \partial_{0 \mid x}^{\beta, \psi}(\eta u)(t, \cdot)\right)(L) .
$$

Using Lemma 4 and the boundary conditions (3), one obtains

$$
\left(I_{0}^{\beta, \psi} \partial_{0 \mid x}^{\beta, \psi}(\eta u)(t, \cdot)\right)(L)=\eta(t, L) u(t, L)-\eta(t, 0) u(t, 0)=0 .
$$

Hence, it holds

$$
\partial_{0 \mid t}^{\alpha, \psi}\left(I_{0}^{\beta, \psi} \eta(t, \cdot)\right)(L)=0
$$

i.e.,

$$
\partial_{0 \mid t}^{\alpha, \psi} \int_{0}^{L} \mathcal{K}_{L}(x)^{\beta-1} \psi^{\prime}(x) \eta(t, x) d x=0
$$

which implies that

$$
I_{0}^{\alpha, \psi} \partial_{0 \mid t}^{\alpha, \psi} \int_{0}^{L} \mathcal{K}_{L}(x)^{\beta-1} \psi^{\prime}(x) \eta(t, x) d x=0 .
$$

Again, using Lemma 4, one deduces that

$$
\int_{0}^{L} \mathcal{K}_{L}(x)^{\beta-1} \psi^{\prime}(x) \eta(t, x) d x-\int_{0}^{L} \mathcal{K}_{L}(x)^{\beta-1} \psi^{\prime}(x) \eta(0, x) d x=0
$$

which yields (25).

Our principal result is the following.

Theorem 1. Suppose that for some $0<T_{0}<\infty,(\eta, u) \in C^{1}(\mathcal{Q}) \times C^{1}(\mathcal{Q}), \mathcal{Q}=\left[0, T_{0}\right] \times[0, L], \eta \geq 0$, is a solution of problem (1)-(2)-(3). Let

$$
T_{\max }:=\sup \left\{\tau>0:(\eta, u) \in C^{1}([0, \tau) \times[0, L]) \times C^{1}([0, \tau) \times[0, L]) \text { is a solution of }(1)-(2)-(3)\right\} .
$$

If

$$
J(0):=\int_{0}^{L} \eta_{0}(x) u_{0}(x) \mathcal{K}_{L}(x)^{\frac{\beta}{2}-1} \psi^{\prime}(x) d x>0
$$


where $\mathcal{K}_{L}$ is given by (26), then

$$
T_{0} \leq T_{\max } \leq \psi^{-1}(\psi(0)+M(a, \alpha))<\infty,
$$

where $M(a, \alpha)$ is given by (11) (with $\theta=\alpha$ ),

$$
a=2 \frac{\Gamma\left(1+\frac{\beta}{2}\right)}{\Gamma\left(1-\frac{\beta}{2}\right)} \frac{\mathcal{K}_{L}(0)^{-\frac{\beta}{2}}}{m_{0}}
$$

and $m_{0}$ is given by (25).

Proof. We introduce the function

$$
\varphi(x)=\mathcal{K}_{L}(x)^{\frac{\beta}{2}-1} \psi^{\prime}(x), \quad 0 \leq x<L .
$$

Multiplying the second equation in (1) by $\varphi(x)$ and integrating over $(0, L)$, one obtains

$$
\begin{aligned}
& \frac{1}{2} \int_{0}^{L} \varphi(x) \frac{1}{\psi^{\prime}(t)} \partial_{t}(\eta u)(t, x) d x+\frac{1}{2} \int_{0}^{L} \varphi(x) \partial_{0 \mid t}^{\alpha, \psi}(\eta u)(t, x) d x+\int_{0}^{L} \varphi(x) \partial_{0 \mid x}^{\beta, \psi}\left(\eta u^{2}\right)(t, x) d x \\
& +\int_{0}^{L} \varphi(x) \partial_{0 \mid x}^{\beta, \psi}\left(\eta^{2}\right)(t, x) d x=0, \quad 0<t<T_{0},
\end{aligned}
$$

which yields

$$
\frac{1}{2 \psi^{\prime}(t)} J^{\prime}(t)+\frac{1}{2}\left(\partial_{0 \mid t}^{\alpha, \psi} J\right)(t)=-\int_{0}^{L} \varphi(x) \partial_{0 \mid x}^{\beta, \psi}\left(\eta u^{2}\right)(t, x) d x-\int_{0}^{L} \varphi(x) \partial_{0 \mid x}^{\beta, \psi}\left(\eta^{2}\right)(t, x) d x, \quad 0<t<T_{0},
$$

where

$$
J(t)=\int_{0}^{L} \varphi(x)(\eta u)(t, x) d x, \quad 0 \leq t \leq T_{0} .
$$

On the other hand, using (8), one has

$$
\int_{0}^{L} \varphi(x) \partial_{0 \mid x}^{\beta, \psi}\left(\eta u^{2}\right)(t, x) d x=\int_{0}^{L}\left(I_{0}^{1-\beta, \psi} \frac{\partial_{x}\left(\eta u^{2}\right)(t, \cdot)}{\psi^{\prime}}\right)(x) \frac{\varphi(x)}{\psi^{\prime}(x)} \psi^{\prime}(x) d x .
$$

Hence, by Lemma 3, one obtains

$$
\begin{aligned}
\int_{0}^{L} \varphi(x) \partial_{0 \mid x}^{\beta, \psi}\left(\eta u^{2}\right)(t, x) d x & =\int_{0}^{L} \frac{\partial_{x}\left(\eta u^{2}\right)(t, x)}{\psi^{\prime}(x)}\left(I_{L}^{1-\beta, \psi} \frac{\varphi}{\psi^{\prime}}\right)(x) \psi^{\prime}(x) d x \\
& =\int_{0}^{L} \partial_{x}\left(\eta u^{2}\right)(t, x)\left(I_{L}^{1-\beta, \psi} \frac{\varphi}{\psi^{\prime}}\right)(x) d x
\end{aligned}
$$

Next, using an integration by parts and the boundary conditions (3), one deduces that

$$
\int_{0}^{L} \varphi(x) \partial_{0 \mid x}^{\beta, \psi}\left(\eta u^{2}\right)(t, x) d x=-\int_{0}^{L} \eta(t, x) u^{2}(t, x) \partial_{x}\left(I_{L}^{1-\beta, \psi} \frac{\varphi}{\psi^{\prime}}\right)(x) d x .
$$

Similarly, one has

$$
\int_{0}^{L} \varphi(x) \partial_{0 \mid x}^{\beta, \psi}\left(\eta^{2}\right)(t, x) d x=-\int_{0}^{L} \eta^{2}(t, x) \partial_{x}\left(I_{L}^{1-\beta, \psi} \frac{\varphi}{\psi^{\prime}}\right)(x) d x .
$$

It follows from (30)-(32) that

$$
\frac{1}{2 \psi^{\prime}(t)} J^{\prime}(t)+\frac{1}{2}\left(\partial_{0 \mid t}^{\alpha, \psi} J\right)(t)=\int_{0}^{L}\left(\eta u^{2}\right)(t, x) \partial_{x}\left(I_{L}^{1-\beta, \psi} \frac{\varphi}{\psi^{\prime}}\right)(x) d x+\int_{0}^{L} \eta^{2}(t, x) \partial_{x}\left(I_{L}^{1-\beta, \psi} \frac{\varphi}{\psi^{\prime}}\right)(x) d x
$$


Next, using (29), for $x \in(0, L)$, an elementary calculation gives us that

$$
\left(I_{L}^{1-\beta, \psi} \frac{\varphi}{\psi^{\prime}}\right)(x)=\frac{\Gamma\left(\frac{\beta}{2}\right)}{\Gamma\left(1-\frac{\beta}{2}\right)} \mathcal{K}_{L}(x)^{-\frac{\beta}{2}} .
$$

Hence, it holds

$$
\partial_{x}\left(I_{L}^{1-\beta, \psi} \frac{\varphi}{\psi^{\prime}}\right)(x)=\frac{\Gamma\left(1+\frac{\beta}{2}\right)}{\Gamma\left(1-\frac{\beta}{2}\right)} \mathcal{K}_{L}(x)^{-\frac{\beta}{2}-1} \psi^{\prime}(x)>0, \quad 0<x<L .
$$

It follows from (33) and (34) that

$$
\frac{1}{2 \psi^{\prime}(t)} J^{\prime}(t)+\frac{1}{2}\left(\partial_{0 \mid t}^{\alpha, \psi} J\right)(t) \geq \int_{0}^{L}\left(\eta u^{2}\right)(t, x) \partial_{x}\left(I_{L}^{1-\beta, \psi} \frac{\varphi}{\psi^{\prime}}\right)(x) d x .
$$

On one hand, by Hölder's inequality, one has

$$
\begin{aligned}
& J^{2}(t) \\
& \leq\left(\int_{0}^{L} \eta(t, x)|u(t, x)| \varphi(x), d x\right)^{2} \\
& =\left(\int_{0}^{L} \sqrt{\eta(t, x)}|u(t, x)| \sqrt{\partial_{x}\left(I_{L}^{1-\beta, \psi} \frac{\varphi}{\psi^{\prime}}\right)(x)} \sqrt{\frac{\eta(t, x)}{\partial_{x}\left(I_{L}^{1-\beta, \psi} \frac{\varphi}{\psi^{\prime}}\right)(x)}} \varphi(x) d x\right)^{2} \\
& \leq\left(\int_{0}^{L}\left(\eta u^{2}\right)(t, x) \partial_{x}\left(I_{L}^{1-\beta, \psi} \frac{\varphi}{\psi^{\prime}}\right)(x) d x\right)\left(\int_{0}^{L} \frac{\eta(t, x)}{\partial_{x}\left(I_{L}^{1-\beta, \psi} \frac{\varphi}{\psi^{\prime}}\right)(x)} \varphi^{2}(x) d x\right) .
\end{aligned}
$$

On the other hand, using (29) and (34), one obtains

$$
\begin{aligned}
& \int_{0}^{L} \frac{\eta(t, x)}{\partial_{x}\left(I_{L}^{1-\beta, \psi} \frac{\varphi}{\psi^{\prime}}\right)(x)} \varphi^{2}(x) d x \\
& =\frac{\Gamma\left(1-\frac{\beta}{2}\right)}{\Gamma\left(1+\frac{\beta}{2}\right)} \int_{0}^{L} \mathcal{K}_{L}(x)^{\beta-1} \psi^{\prime}(x) \eta(t, x) \mathcal{K}_{L}(x)^{\frac{\beta}{2}} d x \\
& \leq \frac{\Gamma\left(1-\frac{\beta}{2}\right)}{\Gamma\left(1+\frac{\beta}{2}\right)} \mathcal{K}_{L}(0)^{\frac{\beta}{2}} \int_{0}^{L} \mathcal{K}_{L}(x)^{\beta-1} \psi^{\prime}(x) \eta(t, x) d x .
\end{aligned}
$$

Furthermore, using the mass conservation law (25), one deduces that

$$
\int_{0}^{L} \frac{\eta(t, x)}{\partial_{x}\left(I_{L}^{1-\beta, \psi} \frac{\varphi}{\psi^{\prime}}\right)(x)} \varphi^{2}(x) d x \leq \frac{\Gamma\left(1-\frac{\beta}{2}\right)}{\Gamma\left(1+\frac{\beta}{2}\right)} \mathcal{K}_{L}(0)^{\frac{\beta}{2}} m_{0} .
$$

Next, (36) and (37) yield

$$
\int_{0}^{L}\left(\eta u^{2}\right)(t, x) \partial_{x}\left(I_{L}^{1-\beta, \psi} \frac{\varphi}{\psi^{\prime}}\right)(x) d x \geq \frac{\Gamma\left(1+\frac{\beta}{2}\right)}{\Gamma\left(1-\frac{\beta}{2}\right)} \frac{\mathcal{K}_{L}(0)^{-\frac{\beta}{2}}}{m_{0}} J^{2}(t), \quad 0<t<T .
$$


It follows from (35) and (38) that

$$
\frac{1}{\psi^{\prime}(t)} J^{\prime}(t)+\left(\partial_{0 \mid t}^{\alpha, \psi} J\right)(t) \geq 2 \frac{\Gamma\left(1+\frac{\beta}{2}\right)}{\Gamma\left(1-\frac{\beta}{2}\right)} \frac{\mathcal{K}_{L}(0)^{-\frac{\beta}{2}}}{m_{0}} J^{2}(t), \quad 0<t<T_{0} .
$$

Hence, using (27) and Lemma 5, the estimate (28) follows.

Example 1. Consider the system

$$
\begin{cases}{ }^{C} D_{0 \mid t}^{\alpha} \eta+{ }^{C} D_{0 \mid x}^{\beta}(\eta u) & =0, \quad t>0,0<x<L, \\ \frac{1}{2}\left[\partial_{t}(\eta u)+{ }^{C} D_{0 \mid t}^{\alpha}(\eta u)\right]+{ }^{C} D_{0 \mid x}^{\beta}\left(\eta u^{2}\right)+{ }^{C} D_{0 \mid x}^{\beta}\left(\eta^{2}\right) & =0, \quad t>0,0<x<L\end{cases}
$$

under the initial and boundary conditions (2) and (3). Here ${ }^{C} D_{0 \mid t}^{\alpha}$ is the Caputo derivative in time of fractional order $0<\alpha<1$ and ${ }^{C} D_{0 \mid x}^{\beta}$ is the Caputo derivative in space of fractional order $0<\beta<1$. System (39) is a special case of (1) with $\psi(s)=s$. Hence, by Theorem 1 , one deduces that if $(\eta, u) \in C^{1}\left(\left[0, T_{0}\right] \times[0, L]\right) \times$ $C^{1}\left(\left[0, T_{0}\right] \times[0, L]\right)$ is a solution of problem (39)-(2)-(3) for some $0<T_{0}<\infty$, and

$$
J(0):=\int_{0}^{L} \eta_{0}(x) u_{0}(x)(L-x)^{\frac{\beta}{2}-1} d x>0,
$$

then

$$
T_{0} \leq T_{\max } \leq M(a, \alpha)<\infty,
$$

where $M(a, \alpha)$ is given by (11) (with $\theta=\alpha$ ) and

$$
a=2 \frac{\Gamma\left(1+\frac{\beta}{2}\right)}{\Gamma\left(1-\frac{\beta}{2}\right)} L^{\frac{-\beta}{2}}\left(\int_{0}^{L}(L-x)^{\beta-1} \eta_{0}(x) d x\right)^{-1} .
$$

\section{Conclusions}

A fractional in time and space shallow-water system is investigated in this paper. The considered fractional derivative depends of a function $\psi \in C^{1}([0, \infty))$, and generalizes Caputo fractional derivative, which corresponds to the case $\psi(t)=t$. Using the test function method, it is shown that under certain conditions imposed on the initial data, the system admits no global in time solutions. Furthermore, an upper bound of the lifespan is obtained.

Author Contributions: Investigation, M.J., M.K. and B.S. M.J., M.K. and B.S. contributed equally to this work.

Funding: M. Jleli is supported by Researchers Supporting Project number (RSP-2019/57), King Saud University, Riyadh, Saudi Arabia. M. Kirane is supported by "RUDN University program 5-100".

Conflicts of Interest: The authors declare no conflict of interest.

\section{References}

1. Mitidieri, E.; Pokhozhaev, S.I. A priori estimates and blow-up of solutions to nonlinear partial differential equations and inequalities. Proc. Steklov Inst. Math. 2011, 234, 1-362.

2. Korpusov, M.O.; Yushkov, E.V. Solution blowup for systems of shallow-water equations. Theor. Math. Phys. 2013, 177, 1505-1514. [CrossRef]

3. Landau, L.D.; Lifshitz, E.M. Fluid Mechanics; Nauka: Moscow, Russia, 1986. (In Russian)

4. Rozhdestvenskij, B.L.; Yanenko, N.N. Systems of Quasilinear Equations and their Applications to Gas Dynamics Series. In Translations of Mathematical Monographs, American Mathematical Society, 2nd ed.; AMS: Providence, RI, USA, 1983; p. 676. 
5. Arshad, S.; Sohail, A.; Maqbool, K. Nonlinear shallow water waves: A fractional order approach. Alex. Eng. J. 2016, 55, 525-532. [CrossRef]

6. Atanackovic, T.; Pilipovic, S.; Stankovic, B.; Zorica, D. Fractional Calculus with Applications in Mechanics: Vibrations and Diffusion Processes; Wiley-ISTE: London, UK, 2014.

7. Hilfer, R. (Ed.) Applications of Fractional Calculus in Physics; World Scientific: Singapore, 1999.

8. Uchaikin, V. Fractional Derivatives for Physicists and Engineers; Springer: Berlin, Germany, 2013.

9. Khater, M.M.; Kumar, D. New exact solutions for the time fractional coupled Boussinesq-Burger equation and approximate long water wave equation in shallow water. J. Ocean Eng. Sci. 2017, 2, 223-228. [CrossRef]

10. Kumar, S. A numerical study for solution of time fractional nonlinear shallow water equation in oceans. Z. Naturforsch. A 2013, 68a, 1-7. [CrossRef]

11. Kumar, D.; Darvishi, M.T.; Joardar, A.K. Modified Kudryashov method and its application to the fractional version of the variety of Boussinesq-like equations in shallow water. Opt. Quantum Electron. 2018, 50, 1-17. [CrossRef]

12. Sahoo, S.; Ray, S.S. New double-periodic solutions of fractional Drinfeld-Sokolov-Wilson equation in shallow water waves. Nonlinear Dyn. 2017, 88, 1869-1882. [CrossRef]

13. Bai, Z.; Chen, Y.; Lian, H.; Sun, S. On the existence of blow up solutions for a class of fractional differential equations. Fract. Calc. Appl. Anal. 2014, 17, 1175-1187. [CrossRef]

14. $\mathrm{Hu}, \mathrm{J} . ; \mathrm{Xin}, \mathrm{J} . ; \mathrm{Lu}, \mathrm{H}$. The global solution for a class of systems of fractional nonlinear Schrödinger equations with periodic boundary condition. Comput. Math. Appl. 2011, 62, 1510-1521. [CrossRef]

15. Kirane, M.; Malik, S.A. The profile of blowing-up solutions to a nonlinear system of fractional differential equations. Nonlinear Anal. Theory Methods Appl. 2010, 73, 3723-3736. [CrossRef]

16. Almeida, R. A Caputo fractional derivative of a function with respect to another function. Commun. Nonlinear Sci. Numer. Simul. 2017, 44, 460-481. [CrossRef]

17. Tao, T. Finite time blowup for an averaged three-dimensional Navier-Stokes equation. J. Am. Math. Soc. 2016, 29, 601-674. [CrossRef]

18. Samko, S.G.; Kilbas, A.A.; Marichev, O.I. Fractional Integrals and Derivatives: Theory and Applications; Gordon and Breach: Yverdon, Switzerland, 1993.

19. Panin, A.A. Local solvability and blowup of the solution of the Rosenau-Bürgers equation with different boundary conditions. Theor. Math. Phys. 2013, 177, 1361-1376. [CrossRef]

(C) 2019 by the authors. Licensee MDPI, Basel, Switzerland. This article is an open access article distributed under the terms and conditions of the Creative Commons Attribution (CC BY) license (http://creativecommons.org/licenses/by/4.0/). 